\title{
Perda de diversidade taxonômica e funcional de aves em área urbana no sul do Brasil
}

\author{
Anne G. Sacco' ${ }^{1}$ Ana Maria Rui², Fabiane B. Bergmann³, Sandra C. Müller' \& Sandra M. Hartz'
}

1. Programa de Pós-Graduação em Ecologia, Instituto de Biociências, Universidade Federal do Rio Grande do Sul. Av. Bento Gonçalves, 9500, Caixa Postal 15007, 91501-970 Porto Alegre, RS, Brasil. (annesacco@gmail.com, sandra.muller@ufrgs.br, sandra.hartz@ufrgs.br)

2. Departamento de Ecologia, Zoologia e Genética, Instituto de Biologia, Universidade Federal de Pelotas, Campus Universitário Capão do Leão,

Caixa Postal 354, 96001-970, Pelotas, RS, Brasil. (ana.rui@ufpel.edu.br)

3. Centro de Ciências Naturais e Exatas, Universidade Federal de Santa Maria, Av. Roraima, s/n², Camobi, $97105-900$ Santa Maria, RS, Brasil.

(fabiberg@yahoo.com.br)

\begin{abstract}
Reduction in taxonomic and functional bird diversity in an urban area in Southern Brazil. Urbanization is a globally process studied and considered one of the most drastic environmental disturb on biodiversity. This study aimed to evaluate how different intensities of urbanization act in the structure of birds in a medium size city inserted in a wetland area. In addition to the traditional metrics richness, abundance and taxonomic composition, it was found as the functional diversity and redundancy of birds are influenced by urban gradient. The urban area studied was the Pelotas city ( $\left.31^{\circ} 46^{\prime \prime} \mathrm{S}, 52^{\circ} 20^{\circ} \mathrm{W}\right)$ situated at the southernmost part of Brazil in a humid region of Pampa biome. A total of 216 fixed observation points along an urbanization gradient were used to sample the avifauna and four independent variables: number of trees, number of buildings with two or more floors, presence of aquatic habitat, and presence of open habitat. Functional traits of species characterized the biomass, diet, forage substrate and nesting substrate. The richness, abundance and functional diversity of avifauna were negatively related to the increase of urban intensity while the redundancy, different from the expected, did not respond to the urban gradient. The traits related to the areas with more intense urbanization (building with two or more floors) were: preference for foraging in the air, omnivory and cavity nesting. Areas with less intense urbanization (more trees and open habitats) were related to the preference for nesting on vegetation and in lower sites, and carnivory. The study showed the importance of the presence of open and aquatic habitats for the avifauna, landscape features typically not cited in urban studies, which highlight the need to adequate the mitigation actions for conservation efforts inside urban habitats according to the regional pool of species.
\end{abstract}

KEYWORDS. Biotic homogenization, Rao's functional diversity, wetlands, Pampa biome.

RESUMO. A urbanização é um processo globalmente estudado e considerado um dos distúrbios mais drásticos sobre a biodiversidade. Este estudo teve como objetivo avaliar como diferentes intensidades de urbanização atuam na estruturação da avifauna em uma cidade de tamanho médio e inserida em região de área úmida. Além das métricas tradicionais de riqueza, abundância e composição taxonômica, verificou-se como a diversidade e redundâncias funcionais das aves são influenciadas pelo gradiente urbano. A área urbana estudada foi a cidade de Pelotas (31 $46^{\prime}$ 'S, $\left.52^{\circ} 20^{\prime} \mathrm{W}\right)$, extremo sul do Brasil, que está inserida em uma área úmida do bioma Pampa. A amostragem das aves e a medida das variáveis urbanas (número de árvores, número de construções de até dois andares ou de mais de dois andares, presença de ambiente aquático e presença de ambiente aberto) foram realizadas em 216 pontos fixos de observação que foram marcados em diferentes intensidades de urbanização. Os atributos funcionais utilizados para caracterizar as espécies foram os relacionados à massa, dieta, substrato de forrageamento e substrato de nidificação. $\mathrm{O}$ aumento da intensidade de urbanização diminuiu a riqueza, a abundância e a diversidade funcional, sendo que a redundância, diferente do esperado, não aumentou com a urbanização. Foi possível observar que os atributos relacionados a áreas com urbanização mais intensa (com contruções de até dois andares e de mais de dois andares) foram: preferência por forrageamento no ar, onivoria e ninhos em cavidades. Os atributos relacionados a áreas com menor intensidade de urbanização e com áreas úmidas foram: preferência de forrageamento na água e de ninhos sobre a água; e aquele relacionados a menores intensidades de urbanização (com maior número de árvores e maior presença de ambientes abertos) foram: preferência por ninhos em vegetação, em locais baixos e no solo e carnivoria. O estudo mostrou a importância da presença de habitats abertos e aquáticos para a avifauna, paisagem tipicamente não citada em estudos urbanos, que destacam a necessidade de adequar as ações de mitigação para os esforços de conservação dentro de habitats urbanos de acordo com o pool regional de espécies.

PALAVRAS-CHAVE. Homogeneização biótica, diversidade funcional de Rao, áreas úmidas, bioma Pampa.

O processo de transformar as áreas naturais em estruturas artificiais para atender as necessidades dos seres humanos é chamado de urbanização e se caracteriza pela presença de grande quantidade de superfícies impermeáveis e alta densidade de pessoas, além da presença de animais e plantas domésticas que substituem as espécies nativas (GILBERT, 1989). A urbanização consiste e m uma das principais causas de mudanças na paisagem (WILCox \& MURPHY, 1985), pois apresenta um estado de permanência, sem retorno da cobertura vegetal original (MARZLUFF \& EwING, 2001; MCKINNEY, 2002). Neste contexto, o desafio fundamental para a conservação é entender os efeitos deste processo sobre a biodiversidade (MCKINNEY, 2002).
Efeitos negativos relacionados à intensidade da urbanização sobre a riqueza foram identificados em diversas áreas urbanas (ver revisão em CHACE \& WALSH, 2006). A urbanização pode promover a perda de espécies através da redução e fragmentação da área disponível para as espécies nativas (McKinneY, 2002), e da simplificação na estrutura vertical da vegetação presente (predomínio de cobertura de gramíneas e herbáceas em detrimento de árvores e arbustos) (MARZLuFf \& EwING, 2001; TOLEDO et al., 2012). Esses fatores combinados reduzem a área $\mathrm{e}$ a qualidade de habitat para animais e tendem a aumentar com a intensidade de urbanização (ALBERTI et al., 2001; HAHS \& McDonNel, 2006). Por outro lado, a urbanização 
pode aumentar a diversidade de espécies, usualmente pela adição de espécies exóticas (McKInNEY, 2002), através da, por exemplo, alta heterogeneidade espacial produzida por diferentes usos do solo em pequenas escalas aliado a ofertas variadas de recursos (SAVARD et al., 2000).

Estudos que tenham avaliado as respostas de diferentes intensidades de urbanização na abundância e na composição das aves são mais escassos em comparação aos estudos que avaliam riqueza de espécies (MARZLUFF, 2001; Chace \& Walsh, 2006; Fontana et al., 2011). A abundância tende a ser maior em níveis intermediários de urbanização (GARAFFA et al., 2009; VILlegas \& Garitano-ZAVALA, 2010) ou apresenta o seu valor máximo em altas intensidades de urbanização (CLERGEAU et al., 1998). Um dos motivos é o aumento na abundância de espécies sinantrópicas (DonNelly \& MARZlufF, 2006), sugerindo que comunidades de aves tornam-se cada vez mais densas, porém empobrecidas à medida que aumenta a intensidade de urbanização. Este padrão, em que poucas espécies com características que as permitem tolerarem o ambiente urbano substituem várias espécies nativas que não toleram as alterações impostas pela urbanização, chamase homogeneização biótica e se repete em várias regiões do mundo (McKinney \& Lockwood, 1999; Olden et al., 2004).

Avaliações acerca das relações entre ecossistemas e biodiversidade quando embasadas somente na riqueza de espécies e abundância de indivíduos são consideradas parciais e pouco preditivas dos processos ecológicos, sendo fundamental considerar aspectos de atributos funcionais (VANDEWAlle et al., 2010). A inserção de atributos funcionais - conjunto de atributos ecológicos importantes para a manutenção das espécies no ambiente - no estudo de comunidades permite, por exemplo, determinar como a composição das características funcionais varia entre assembleias de espécies ao longo de gradientes espaciais (STEVEns et al., 2003) ou temporais (Micheli \& HALPERN, 2005), e entender por que algumas espécies se tornam abundantes ou raras em ambientes distintos.

A urbanização pode ser determinante em alterar ou mesmo suprimir alguns atributos funcionais de assembleias de aves, já que engloba vários fatores de modificação de habitat (KARK et al., 2007; FLYNN et al., 2009). Conforme BlaIR (1996) sugeriu, espécies que conseguem ultrapassar o filtro ambiental imposto pela urbanização intensa podem ser chamadas de "tolerantes urbanos" em contraponto aos "intolerantes urbanos", cujos atributos são incompatíveis com estes ambientes. Assim, mudanças nos atributos podem ser avaliadas em termos de composição nas diferenças ecológicas entre espécies. Índices de diversidade funcional expressam a variabilidade local em termos de atributos funcionais das espécies presentes e, muitas vezes, estão fortemente relacionados à riqueza de espécies - especialmente quando os atributos das espécies presentes forem complementares (Petchey \& Gaston, 2002; Mouchet et al., 2010). Neste sentido, avaliações sobre a redundância funcional também devem ser consideradas, já que são informativas de como uma espécie pode ou não compensar a perda de outra em ambientes impactados, dadas as suas semelhanças em termos de atributos funcionais (PETCHeY et al., 2009).

Este estudo teve como objetivo avaliar como as características de um ambiente urbano e as diferentes intensidades de urbanização atuam na estruturação da avifauna em uma cidade considerada de tamanho médio (até 500 mil habitantes; IPEA, 2008) e inserida em região de área úmida. Foram testadas as seguintes hipóteses: (1) a riqueza e abundância de espécies serão influenciadas, respectivamente, de modo negativo e positivo pelo gradiente de urbanização; (2) a composição taxonômica sofrerá uma mudança ao longo do gradiente de urbanização, com impacto mais pronunciado sobre as espécies de áreas úmidas e (3) a diversidade funcional diminuirá conforme aumenta o gradiente de urbanização, enquanto que a redundância ecológica tenderá a aumentar, dada a potencial homogeneização da biota nos centros urbanos.

\section{MATERIAL E MÉTODOS}

Área de estudo. $O$ estudo foi realizado no município de Pelotas, situado no extremo sul do Brasil (31 ${ }^{\circ} 46^{\prime} \mathrm{S}$, $52^{\circ} 20^{\prime} \mathrm{W}$ ). A população está estimada em mais de 320 mil habitantes, sendo $93 \%$ de sua população residente na zona urbana (IBGE, 2010). Pelotas encontra-se localizada na Planície Costeira a sete metros acima do nível do mar, às margens da Lagoa dos Patos, que é a maior lagoa do Brasil, com uma área de $10.360 \mathrm{~km}^{2}$ (CALLIARI, 1998). Está inserida no Bioma Pampa, na fisionomia das Formações Pioneiras (vegetação com influência fluvial e/ou lacustre) (IBGE, 1992). Atualmente, somente $16,17 \%$ da cobertura vegetal original do Pampa no município permanecem intactas, sendo o restante modificado para algum tipo de uso antrópico (PROBIO, 2007). Mais informações sobre a cidade de Pelotas e um mapa da área de estudo podem ser vistos em SACCO et al. (2013).

Desenho amostral e coleta de dados. A coleta de dados ocorreu de outubro de 2007 a setembro de 2008. As aves foram amostradas através do método do ponto fixo de contagem (BibBy et al., 1992), com um tempo de permanência de oito minutos, um raio fixo de observação de $50 \mathrm{~m}$ e uma distância de no mínimo $200 \mathrm{~m}$ entre os pontos para evitar contagens duplas de indivíduos e garantir a independência entre as unidades amostrais. Foram distribuídos 216 pontos fixos de contagem na área urbana e periurbana, de maneira que diferentes intensidades de urbanização fossem amostradas. Estes foram divididos em grupos de 12 pontos conforme a proximidade, considerando que a distância entre um ponto e outro era percorrida a pé, sendo sorteados ao longo dos dias de amostragem. Setenta e dois pontos foram amostrados por mês (12 a cada dia), no período da manhã, iniciando por volta de 30 minutos após o amanhecer, com duração em torno de duas horas e meia. A sequência dos pontos era alternada para que estes não fossem amostrados sempre no mesmo horário. 
Desta forma, a totalidade dos pontos foi amostrada uma vez a cada três meses, sendo então reamostrados quatro vezes, em diferentes estações do ano. Os registros das aves foram visuais, com auxílio de binóculo, ou auditivas, sendo contabilizado o número de indivíduos de cada espécie. Apenas os indivíduos de Passer domesticus (Linnaeus, 1758) não foram contabilizados, já que é uma espécie extremamente abundante em ambiente urbano, o que torna difícil sua contagem.

As variáveis ambientais foram mensuradas nos mesmos pontos fixos de contagem das aves, e também num raio de $50 \mathrm{~m}$, sendo as seguintes: a) número de árvores: indivíduos com mais de 1,50 m de altura; b) presença de áreas úmidas: banhados, lagoas, lagos, rios ou canais; c) presença de ambientes abertos: campos e terrenos baldios, ambientes com vegetação rasteira sem construções; d) número de construções com até dois andares; e) número de construções com mais de dois andares.

Análise dos dados. Inicialmente, para verificar se havia uma correlação espacial entre os pontos amostrais, uma análise de correlação de Mantel (MANTEL, 1967) foi realizada entre uma matriz de distância feita a partir da matriz de composição taxonômica (dissimilaridade de Bray-Curtis) e uma matriz de distância feita a partir das coordenadas geográficas de cada ponto. O teste, realizado no software Multiv (Pillar, 2006), indicou que as unidades amostrais foram estatisticamente independentes, não havendo correlação espacial significativa $(\mathrm{p}=0,096)$.

Espécies com menos de cinco indivíduos ou registradas em somente uma das quatro amostragens foram desconsiderados nas análises subsequentes. Para as análises de abundância, foi utilizada a média de indivíduos de cada espécie contabilizados nas quatro amostragens realizadas ao longo do ano, a fim de evitar superamostragem das espécies (ou seja, contagem dos mesmos indivíduos). Para avaliação do padrão de riqueza, foram utilizados os resíduos da regressão entre número de espécies e abundância de indivíduos por ponto, uma vez que o número de espécies foi influenciado pelo número de indivíduos registrados $\left(\mathrm{R}^{2}=0,36 ; \mathrm{p}<0,001\right)$.

As variáveis ambientais foram sintetizadas a partir de uma Análise de Componentes Principais (PCA) (baseada em correlação entre as variáveis, através do software Multiv, PILlAR, 2006), de modo a expressar a maior variação na complexidade estrutural do ambiente urbano. O eixo 1 desta ordenação (com $40 \%$ de explicação) foi utilizado como indicador do gradiente de intensidade de urbanização (maior nos escores maiores) para posteriormente avaliar a relação com a diversidade taxonômica e funcional das aves. Conforme a Fig. 1, vemos que a intensidade de urbanização está sendo expressa pelo aumento na quantidade de prédios e diminuição no número de árvores e na proporção de áreas abertas e úmidas. O eixo 2 também está relacionado ao ambiente urbano, porém numa proporção menor (19,5\% da variação total), sendo que os maiores escores indicam um aumento no número de construções de até dois andares.

Cada espécie de ave registrada foi caracterizada com

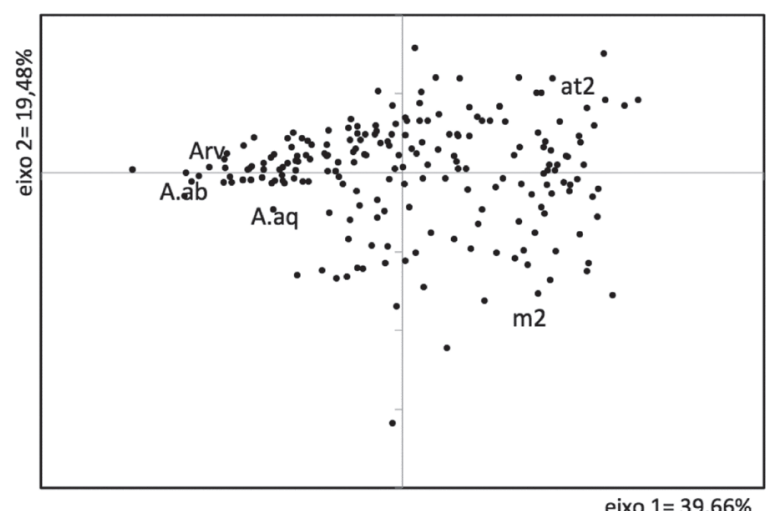

Fig. 1. Diagrama de dispersão das unidades amostrais descritas pelas variáveis ambientais, representada pelo primeiro e pelo segundo eixo de ordenação (PCA), amostradas na cidade de Pelotas, RS, Brasil durante o período de outubro de 2007 a setembro de 2008 (Rótulos das variáveis: A.Aq, presença de ambiente aquático; A.ab, presença de ambientes abertos; Arv, número de árvores; $\mathrm{m} 2$, número de construções com mais de dois andares; at2, número de construções com até dois andares).

atributos funcionais relacionados com potenciais respostas das espécies à variação na estrutura do habitat. Assim, as espécies foram caracterizadas por: massa corpórea, dieta (onívora, insetívora, carnívora, frugívora, granívora, nectarívora), substrato de forrageamento (solo, água, ar, vegetação) e substrato de nidificação (cavidades, vegetação, solo, sobre a água). Os atributos foram definidos com informações retiradas da literatura (BELTon, 1994; STOTZ et al., 1996; Sick, 1997; Del Hoyo et al., 1992-2002, 2003 2006; KRÜGEl \& ANJos, 2000; ANJos, 2001) (Tab. I). Os atributos qualitativos foram expandidos e transformados em variáveis binárias (variáveis dummy) para permitir análises quantitativas (PILlaR et al., 2009).

Para testar a primeira hipótese deste estudo - relação à riqueza e abundância contra o gradiente de urbanização foram realizadas análises de regressões lineares utilizando os escores do eixo 1 da PCA (medida que representou o gradiente de intensidade de urbanização) sobre a riqueza e abundância (valores em escala log) das aves. Para testar a segunda hipótese deste estudo - a influência das variáveis ambientais na composição taxonômica das aves - foi realizada uma análise de correspondência canônica (CCA, TER BRAAK, 1986). Para analisar a terceira hipótese, foi calculada a diversidade funcional (DF) para cada ponto amostral a partir da entropia quadrática de Rao (RAO, 1982), baseada numa matriz de dissimilaridade utilizando a distância de Gower (Gower, 1971). A DF foi obtida considerando separadamente os grupos de atributos que contêm relações funcionais (por exemplo, dieta), assim como para todos os atributos reunidos (diversidade funcional total), através do software Syncsa 2.6.9 (por V. D. Pillar, disponível em http://ecoqua.ecologia.ufrgs.br). Além disso, a redundância funcional (RF) foi calculada segundo DE BELLo et al. (2007), entendida como sendo a diferença entre a diversidade taxonômica (calculada a partir do índice de Gini-Simpson) e a funcional (Rao) (PILlar et al., 2013). A seguir, análises de regressões lineares foram 
Tab. I. Lista taxonômica (CBRO, 2014) das 67 espécies de aves utilizadas para análise e registradas na área urbana do município de Pelotas, Rio Grande do Sul, Brasil, com o número médio de indivíduos de cada espécie obtido em quatro períodos amostrais entre os meses de outubro de 2007 a setembro de 208 e a classificação das espécies segundo massa corpórea, dieta, substrato de forrageamento e substrato de nidificação (Dieta: He, herbívoros; On, onívoros; In, insetívoros; Car, carnívoros; Gr, granívoros; Fr, frugívoros; Ne, nectarívoros. Substrato de forrageamento: So, solo; Ag, água; Vg, vegetação; Ar, ar). Substrato de nidificação (So, solo; S.A, sobre a água; Vg, vegetação; Cv, cavidades; Ba, baixo; Al, alto ).

\begin{tabular}{|c|c|c|c|c|c|}
\hline Táxons & $\begin{array}{l}\text { Abundância } \\
\text { média }\end{array}$ & $\begin{array}{c}\text { Massa corporal } \\
(\mathrm{g})\end{array}$ & Dieta & $\begin{array}{c}\text { Substrato de } \\
\text { forrageamento }\end{array}$ & $\begin{array}{c}\text { Substrato de } \\
\text { nidificação }\end{array}$ \\
\hline \multicolumn{6}{|l|}{ Anseriformes } \\
\hline \multicolumn{6}{|l|}{ Anhimidae } \\
\hline Chauna torquata (Oken, 1816) & 1,25 & 4400 & $\mathrm{He}$ & So & Vg/S.A \\
\hline \multicolumn{6}{|l|}{ Anatidae } \\
\hline Dendrocygna viduata (Linnaeus, 1766) & 5,75 & 1500 & On & $\mathrm{So} / \mathrm{Ag}$ & So \\
\hline Anas flavirostris (Vieillot, 1816) & 0,75 & 400 & $\mathrm{He}$ & $\mathrm{Ag}$ & $\mathrm{Cv} / \mathrm{So}$ \\
\hline Anas versicolor (Vieillot, 1816) & 3,25 & 400 & $\mathrm{He}$ & $\mathrm{Ag}$ & So \\
\hline \multicolumn{6}{|l|}{ Pelecaniiformes } \\
\hline \multicolumn{6}{|l|}{ Ardeidae } \\
\hline Bubulcus ibis (Linnaeus, 1758) & 4,5 & 350 & In & So & $\mathrm{Vg} / \mathrm{Ba}$ \\
\hline Ardea cocoi (Linnaeus, 1766) & 0,25 & 3200 & Car & $\mathrm{So} / \mathrm{Ag}$ & $\mathrm{Vg} / \mathrm{Al}$ \\
\hline Syrigma sibilatrix (Temminck, 1824) & 6,5 & 370 & In & So & $\mathrm{Vg} / \mathrm{Al}$ \\
\hline Egretta thula (Molina, 1782) & 8 & 500 & Car & So/Ag & $\mathrm{Vg} / \mathrm{Ba}$ \\
\hline \multicolumn{6}{|l|}{ Threskioniithidae } \\
\hline Plegadis chihi (Vieillot, 1817) & 26,75 & 610 & On & $\mathrm{So} / \mathrm{Ag}$ & Vg/S.A \\
\hline Phimosus infuscatus (Lichtenstein, 1823) & 14 & 575 & On & So & $\mathrm{Vg} / \mathrm{Ba}$ \\
\hline \multicolumn{6}{|l|}{ Cathartiformes } \\
\hline \multicolumn{6}{|l|}{ Cathartidae } \\
\hline Cathartes aura (Linnaeus, 1758) & 4,5 & 1500 & Car & $\mathrm{So} / \mathrm{Ar}$ & $\mathrm{Al}$ \\
\hline Coragyps atratus (Bechstein, 1793) & 1 & 1600 & Car & So/Ar & $\mathrm{Al}$ \\
\hline \multicolumn{6}{|l|}{ Accipitriformes } \\
\hline \multicolumn{6}{|l|}{ Accipitridae } \\
\hline Rupornis magnirostris (Gmelin, 1788) & 1 & 284 & Car & $\mathrm{Vg}$ & $\mathrm{Vg} / \mathrm{Al}$ \\
\hline \multicolumn{6}{|l|}{ Gruiformes } \\
\hline \multicolumn{6}{|l|}{ Rallidae } \\
\hline Gallinula galeata (Lichtenstein, 1818) & 1,25 & 300 & On & So/Ar & $\mathrm{Vg} / \mathrm{Ba}$ \\
\hline \multicolumn{6}{|l|}{ Charadriiformes } \\
\hline \multicolumn{6}{|l|}{ Charadriidae } \\
\hline Vanellus chilensis (Molina, 1782) & 106,25 & 277 & In & So & So \\
\hline \multicolumn{6}{|l|}{ Recurvirostridae } \\
\hline Himantopus melanurus (Vieillot, 1817) & 5,5 & 190 & Car & So & So \\
\hline Jacanidae & & & & & \\
\hline Jacana jacana (Linnaeus, 1766) & 4 & 155 & On & So & S.A \\
\hline Laridae & & & & & \\
\hline Chroicocephalus maculipennis (Lichtenstein, 1823) & 23,5 & 320 & On & $\mathrm{So} / \mathrm{Ag}$ & So \\
\hline Chroicocephalus cirrocephalus (Vieillot, 1818) & 18,75 & 288 & On & So/Ag & So/S.A \\
\hline Sternidae & & & & & \\
\hline Phaetusa simplex (Gmelin, 1789) & 7 & 247 & On & $\mathrm{Ag}$ & $\mathrm{Cv} / \mathrm{So}$ \\
\hline Sterna trudeaui (Audubon, 1838) & 1,25 & 150 & On & $\mathrm{Ag}$ & $\mathrm{Vg} / \mathrm{S} . \mathrm{A}$ \\
\hline Columbiformes & & & & & \\
\hline Columbidae & & & & & \\
\hline Columbina talpacoti (Temminck, 1811) & 41,5 & 50 & $\mathrm{Gr}$ & So & Al \\
\hline Columbina picui (Temminck, 1813) & 86,25 & 47 & $\mathrm{Gr}$ & So & $\mathrm{Vg}$ \\
\hline Columba livia (Gmelin, 1789) & 294 & 300 & On & $\mathrm{So} / \mathrm{Vg}$ & - \\
\hline Patagioenas picazuro (Temminck, 1813) & 0,75 & 400 & $\mathrm{Fr}$ & $\mathrm{Vg}$ & $\mathrm{Vg}$ \\
\hline Zenaida auriculata (Des Murs, 1847) & 14,75 & 130 & $\mathrm{Gr}$ & $\mathrm{So} / \mathrm{Vg}$ & $\mathrm{Vg} / \mathrm{Ba}$ \\
\hline Leptotila verreauxi (Bonaparte, 1855) & 9 & 200 & $\mathrm{Fr}$ & So/Vg & $\mathrm{Vg} / \mathrm{Ba}$ \\
\hline Cuculiformes & & & & & \\
\hline Cuculidae & & & & & \\
\hline Guira guira (Gmelin, 1788) & 44,25 & 140 & In & So & $\mathrm{Vg} / \mathrm{Al}$ \\
\hline Strigiformes & & & & & \\
\hline Strigidae & & & & & \\
\hline Athene cunicularia (Molina, 1782) & 0,75 & 200 & In & So & $\mathrm{Cv} / \mathrm{So}$ \\
\hline Apodiformes & & & & & \\
\hline Trochilidae & & & & & \\
\hline Florisuga fusca (Vieillot, 1817) & 1,5 & 9 & $\mathrm{Ne}$ & $\mathrm{Vg}$ & $\mathrm{Vg} / \mathrm{Al}$ \\
\hline Hylocharis chrysura (Shaw, 1812) & 20 & 5 & $\mathrm{Ne}$ & $\mathrm{Vg}$ & $\mathrm{Vg} / \mathrm{Al}$ \\
\hline Leucochloris albicollis (Vieillot, 1818) & 1,75 & 6 & $\mathrm{Ne}$ & $\mathrm{Vg}$ & $\mathrm{Vg} / \mathrm{Ba}$ \\
\hline Piciformes & & & & & \\
\hline Picidae & & & & & \\
\hline Colaptes melanochloros (Gmelin, 1788) & 3,5 & 130 & In & So/Vg & $\mathrm{Cv} / \mathrm{Vg}$ \\
\hline
\end{tabular}


Tab. I. Cont

\begin{tabular}{|c|c|c|c|c|c|}
\hline Táxons & $\begin{array}{c}\text { Abundância } \\
\text { média }\end{array}$ & $\begin{array}{l}\text { Massa corporal } \\
\text { (g) }\end{array}$ & Dieta & $\begin{array}{c}\text { Substrato de } \\
\text { forrageamento }\end{array}$ & $\begin{array}{c}\text { Substrato de } \\
\text { nidificação }\end{array}$ \\
\hline Colaptes campestris (Vieillot, 1818) & 12,25 & 160 & In & $\mathrm{So} / \mathrm{Vg}$ & $\mathrm{Cv} / \mathrm{Vg}$ \\
\hline \multicolumn{6}{|l|}{ Falconiformes } \\
\hline \multicolumn{6}{|l|}{ Falconidae } \\
\hline Caracara plancus (Miller, 1777) & 6 & 1250 & Car & So & $\mathrm{Vg} / \mathrm{So} / \mathrm{Ba}$ \\
\hline Milvago chimachima (Vieillot, 1816) & 1 & 270 & Car & So/Vg & $\mathrm{Vg} / \mathrm{Al}$ \\
\hline Milvago chimango (Vieillot, 1816) & 5,25 & 260 & Car & $\mathrm{So} / \mathrm{Vg}$ & $\mathrm{Vg} / \mathrm{Al}$ \\
\hline Falco sparverius (Linnaeus, 1758) & 0,75 & 115 & Car & So/ $\mathrm{Vg}$ & $\mathrm{Al}$ \\
\hline \multicolumn{6}{|l|}{ Psittaciformes } \\
\hline \multicolumn{6}{|l|}{ Psittacidae } \\
\hline Myiopsitta monachus (Boddaert, 1783) & 304,5 & 130 & $\mathrm{Fr}$ & $\mathrm{Vg}$ & $\mathrm{Vg} / \mathrm{Al}$ \\
\hline \multicolumn{6}{|l|}{ Passeriformes } \\
\hline \multicolumn{6}{|l|}{ Furnariidae } \\
\hline Furnarius rufus (Gmelin, 1788) & 252,75 & 65 & In & So & $\mathrm{Al}$ \\
\hline \multicolumn{6}{|l|}{ Rhynchocyclidae } \\
\hline Phylloscartes ventralis (Temminck, 1824) & 1,25 & 8 & In & $\mathrm{Vg}$ & $\mathrm{Vg} / \mathrm{Al}$ \\
\hline \multicolumn{6}{|l|}{ Tyrannidae } \\
\hline Pitangus sulphuratus (Linnaeus, 1766) & 432,75 & 75 & On & $\mathrm{So} / \mathrm{Vg}$ & $\mathrm{Al}$ \\
\hline Machetornis rixosa (Vieillot, 1819) & 76 & 35 & In & So & $\mathrm{Cv}$ \\
\hline Tyrannus savana (Vieillot, 1808) & 14,5 & 30 & In & $\mathrm{Vg}$ & Vg \\
\hline Xolmis irupero (Vieillot, 1823) & 4,75 & 30 & In & So/Vg & $\mathrm{Vg} / \mathrm{Al}$ \\
\hline \multicolumn{6}{|l|}{ Vireonidae } \\
\hline Cyclarhis gujanensis (Gmelin, 1789) & 1,25 & 30 & In & $\mathrm{Vg}$ & $\mathrm{Vg} / \mathrm{Al}$ \\
\hline \multicolumn{6}{|l|}{ Hirundinidae } \\
\hline Pygochelidon cyanoleuca (Vieillot, 1817) & 117,25 & 13 & In & $\mathrm{Ar}$ & $\mathrm{Cv}$ \\
\hline Progne tapera (Vieillot, 1817) & 3,5 & 40 & In & $\mathrm{Ar}$ & $\mathrm{Cv}$ \\
\hline Tachycineta leucorrhoa (Vieillot, 1817) & 26,25 & 20 & In & $\mathrm{Ar}$ & $\mathrm{Cv}$ \\
\hline \multicolumn{6}{|l|}{ Troglodytidae } \\
\hline Troglodytes musculus (Naumann, 1823) & 60,75 & 12 & On & So/Vg & $\mathrm{Cv}$ \\
\hline \multicolumn{6}{|l|}{ Turdidae } \\
\hline Turdus rufiventris (Vieillot, 1818) & 29 & 80 & On & So/Vg & $\mathrm{Vg} / \mathrm{Ba}$ \\
\hline Turdus amaurochalinus (Cabanis, 1850) & 13,5 & 70 & On & $\mathrm{So} / \mathrm{Vg}$ & $\mathrm{Vg} / \mathrm{Ba}$ \\
\hline \multicolumn{6}{|l|}{ Mimidae } \\
\hline Mimus saturninus (Lichtenstein, 1823) & 1,5 & 80 & On & $\mathrm{Vg}$ & $\mathrm{Vg} / \mathrm{Al}$ \\
\hline \multicolumn{6}{|l|}{ Passerellidae } \\
\hline Zonotrichia capensis (Statius Muller, 1776) & 8,75 & 25 & On & So/Vg & So \\
\hline \multicolumn{6}{|l|}{ Parulidae } \\
\hline Setophaga pitiayumi (Vieillot, 1817) & 2,25 & 7 & In & $\mathrm{Vg}$ & $\mathrm{Vg} / \mathrm{Al}$ \\
\hline Basileuterus culicivorus (Deppe, 1830) & 4,5 & 10 & In & $\mathrm{Vg}$ & So \\
\hline Myiothlypis leucoblephara (Vieillot, 1817) & 1,5 & 15 & In & $\mathrm{Vg}$ & So \\
\hline \multicolumn{6}{|l|}{ Icteridae } \\
\hline Icterus cayanensis (Linnaeus, 1766) & 1 & 32 & On & $\mathrm{Vg}$ & $\mathrm{Vg}$ \\
\hline Chrysomus ruficapillus (Vieillot, 1819) & 42 & 39 & On & $\mathrm{So} / \mathrm{Vg}$ & $\mathrm{Vg} / \mathrm{Ba}$ \\
\hline Pseudoleistes guirahuro (Vieillot, 1819) & 2,25 & 75 & On & So & $\mathrm{Vg} / \mathrm{Ba}$ \\
\hline Agelaioides badius (Vieillot, 1819) & 3,75 & 45 & On & $\mathrm{Vg}$ & $\mathrm{Al}$ \\
\hline Molothrus bonariensis (Gmelin, 1789) & 1,25 & 55 & On & So & So/Vg \\
\hline \multicolumn{6}{|l|}{ Thraupidae } \\
\hline Coereba flaveola (Linnaeus, 1758) & 9,5 & 10 & $\mathrm{Ne}$ & $\mathrm{Vg}$ & $\mathrm{Vg}$ \\
\hline Tangara sayaca (Linnaeus, 1766) & 59,95 & 34 & $\mathrm{Fr}$ & $\mathrm{Vg}$ & $\mathrm{Vg}$ \\
\hline Paroaria coronata (Miller, 1776) & 1,75 & 43 & Gr & So/Vg & So \\
\hline Pipraeidea bonariensis (Gmelin, 1789) & 1,25 & 42 & $\mathrm{Fr}$ & $\mathrm{Vg}$ & $\mathrm{Vg} / \mathrm{Al}$ \\
\hline Sicalis flaveola (Linnaeus, 1766) & 13,75 & 17 & Gr & So & $\mathrm{Cv}$ \\
\hline
\end{tabular}

realizadas para verificar como a diversidade e a redundância funcional foram influenciadas pelo gradiente de intensidade de urbanização. Para avaliação da composição funcional, primeiramente foram gerados valores médios dos atributos nas comunidades, ponderados pelas abundâncias das espécies em cada unidade amostral (CWM, community weighted mean traits, que corresponde à matriz $\mathrm{T}$, segundo PILlar et al., 2009). Com esta matriz também aplicamos uma CCA, com software Multiv (PILlar, 2006), com o objetivo de verificar a influência do ambiente urbano sobre a composição funcional (atributos) da assembleia de aves.

\section{RESULTADOS}

Foram registradas no total 84 espécies, sendo que destas, 67 espécies de aves foram consideradas nas análises (somente aquelas registradas em mais de uma campanha de amostragem e mais de cinco indivíduos) (Tab. I). A lista completa das espécies registradas, bem como suas respectivas abundâncias sazonais obtidas estão apresentadas em SACCO et al. (2013).

Tanto a riqueza como a abundância foram influenciadas pelo gradiente de intensidade de urbanização 
(Fig. 2). Conforme há um aumento na intensidade de urbanização, a riqueza e a abundância diminuem significativamente.

A CCA das espécies de aves indicou que existe relação significativa (com $44 \%$ de explicação total; $\mathrm{p}=$ 0,001 ) entre a composição e o gradiente de intensidade de urbanização (Fig. 3). O eixo 1 expressa 21,2\% da variação explicada e o eixo 2 representa $10,4 \%$ da variação na composição taxonômica definida pelas variáveis ambientais. Como esperado, uma boa parte das espécies estiveram relacionadas aos ambientes úmidos, abertos e arbóreos, enquanto apenas algumas espécies aos ambientes com presença de construções. Algumas espécies mais abundantes, como Furnarius rufus (Gmelin, 1788), Columba livia (Gmelin, 1789), Myiopsitta monachus (Boddaert, 1783) e Pitangus sulphuratus (Linnaeus, 1766) estiveram associadas a construções com até dois andares, sendo que apenas Columba livia esteve associada com prédios de mais de dois andares. Dentre as espécies de áreas úmidas, apenas Syrigma sibilatrix (Temminck, 1824) esteve associada também a ambientes urbanos.

$\mathrm{O}$ aumento na intensidade de urbanização afetou negativamente tanto a diversidade taxonômica $\left(\mathrm{R}^{2}=0,18\right.$; $\left.\mathrm{F}_{\text {calc }}=46,1 ; \mathrm{gl}=214 ; \mathrm{p}<0,001\right)$, quanto a funcional $\left(\mathrm{R}^{2}=\right.$ 0,$\left.24 ; \mathrm{F}_{\text {calc }}=68,7 ; \mathrm{gl}=214 ; \mathrm{p}<0,001\right)$ (Fig. 4). Apesar de significativa, a relação entre a intensidade de urbanização com a redundância foi de menor intensidade $\left(\mathrm{R}^{2}=0,03 ; \mathrm{F}_{\text {calc }}\right.$ $=6,7 ; \mathrm{gl}=214 ; \mathrm{p}=0,01)$. Quando os conjuntos de atributos foram analisados separadamente (Fig. 5), verificamos que a diversidade funcional relacionada ao substrato de nidificação $\left(\mathrm{R}^{2}=0,31 ; \mathrm{F}_{\text {calc }}=94,2 ; \mathrm{gl}=214 ; \mathrm{p}<0,001\right)$ foi a mais influenciada pela intensidade de urbanização, demonstrando uma queda significativa à medida que a urbanização se torna mais acentuada. Por outro lado, a redundância funcional relacionada a este conjunto de atributos foi a única que não apresentou relação significativa com o gradiente de urbanização (Fig. 5d; $\mathrm{R}^{2}<0,001 ; \mathrm{F}_{\text {calc }}$
$=0,1 ; \mathrm{gl}=214 ; \mathrm{p}=0,68)$. Os índices relacionados aos atributos massa corpórea e tipo de dieta apresentaram padrões distintos, enquanto num a redundância foi mais afetada (massa corpórea: $\mathrm{R}^{2}=0,12 ; \mathrm{F}_{\text {calc }}=30,6 ; \mathrm{gl}=214 ; \mathrm{p}<$ 0,001 ; diversidade funcional: $\mathrm{R}^{2}=0,03 ; \mathrm{F}_{\text {calc }}=7,3 ; \mathrm{gl}=214$; $\mathrm{p}=0,009)$ noutro a diversidade funcional respondeu mais acentuadamente (dieta: $\mathrm{R}^{2}=0,15 ; \mathrm{F}_{\text {calc }}=38,1 ; \mathrm{gl}=214 ; \mathrm{p}<$ 0,001 ; redundância funcional: $\mathrm{R}^{2}=0,02 ; \mathrm{F}_{\text {calc }}=5,2 ; \mathrm{gl}=214$; $\mathrm{p}=0,03)$. Apesar de significativas, as relações dos índices que expressam atributos de substrato de forrageamento representam mudanças menos acentuadas em relação ao gradiente de urbanização (diversidade funcional: $\mathrm{R}^{2}=0,02$, $\mathrm{F}_{\text {calc }}=5,1 ; \mathrm{gl}=214 ; \mathrm{p}=0,03$; redundância funcional: $\mathrm{R}^{2}=$ $\left.0,07, \mathrm{~F}_{\text {calc }}=16,4 ; \mathrm{gl}=214 ; \mathrm{p}=0,002\right)$.

ACCA da composição funcional das aves também foi significativa ( $\mathrm{P}=0,001$, com $14,3 \%$ de explicação total) e mostra a separação pelo eixo 1 ( $65 \%$ de explicação) dos atributos funcionais das aves que são mais representativos em locais de maior intensidade de urbanização, em oposição a locais associados a áreas úmidas (Fig. 6). O eixo 2 ( $24 \%$ de explicação) da CCA expressa uma separação principalmente relacionada aos atributos associados a ambientes urbanos com maior número de árvores ou áreas abertas, em contraponto aos mais associados à intensidade de urbanização ou mesmo às áreas úmidas. Dentre os atributos mais relacionados à áreas com urbanização mais intensa estão a preferência por forrageamento no ar, onivoria e ninhos em cavidades. Já os atributos das aves que estão relacionados à menor intensidade de urbanização e presença de ambientes aquáticos foram maior massa corpórea, preferência de forrageamento na água e de ninhos sobre a água. Os atributos relacionados à menor intensidade de urbanização e maior número de árvores e/ ou maior presença de ambientes abertos foram preferência por ninhos em vegetação, ou em locais baixos e no solo, assim como a carnivoria.
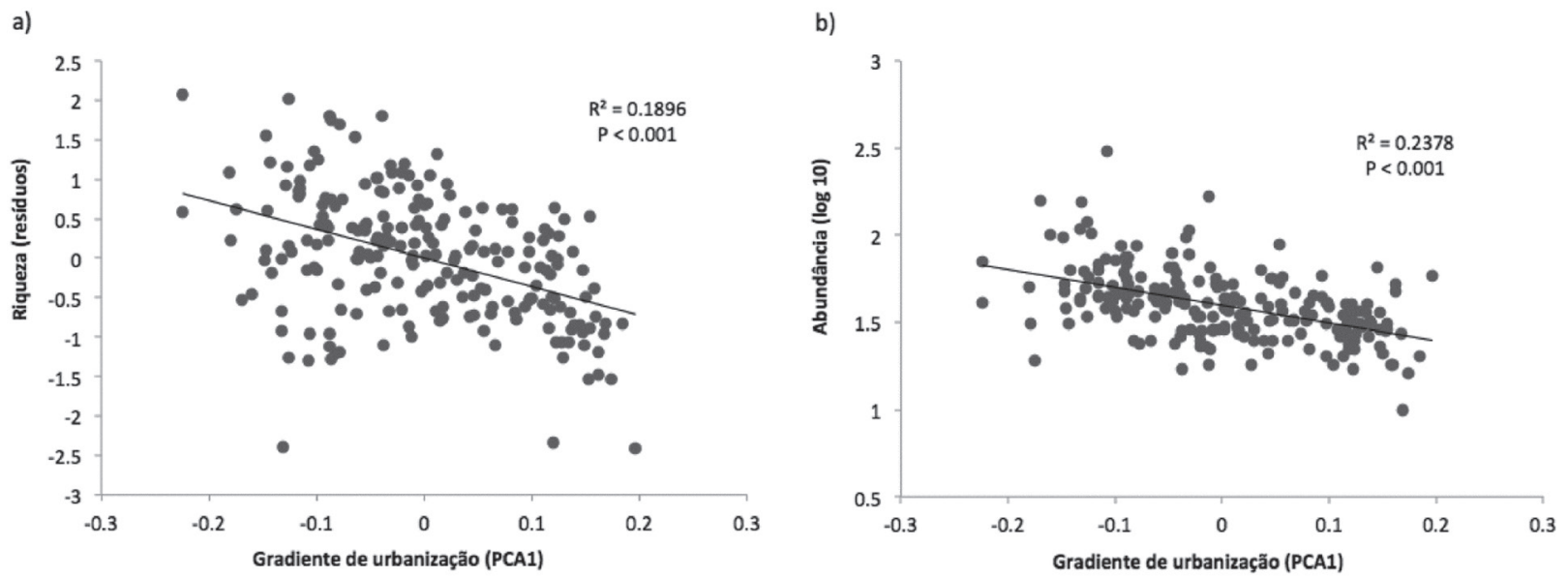

Fig. 2. Influência do gradiente de intensidade de urbanização (escores do eixo 1 da PCA; Fig. 1) na riqueza de espécies (a) (considerando os resíduos de sua relação com a abundância de indivíduos) e na abundância (b) de aves registradas na cidade de Pelotas, RS, Brasil durante o período de outubro de 2007 a setembro de 2008 . 


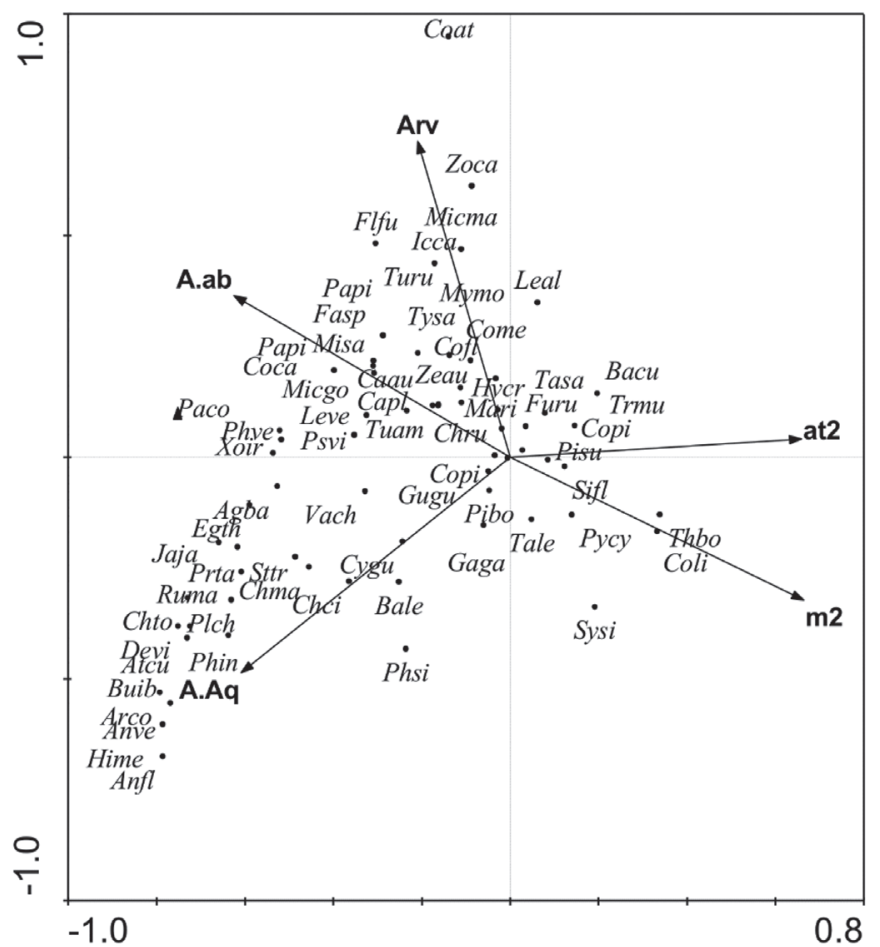

Fig. 3. Diagrama de ordenação definido pela análise de correspondência canônica (CCA) da composição taxonômica de aves em relação às variáveis ambientais associadas com a urbanização de Pelotas, RS, Brasil no período de 2007 a 2008. As setas representam as variáveis ambientais e os pontos representam as espécies de aves [Espécies: Agba (Agelaioides badius); Anfl (Anas flavirostris); Anve (Anas versicolor); Arco (Ardea cocoi); Atcu (Athene cunicularia); Bacu (Basileuterus culicivorus); Bale (Myiothlypis leucoblepharus); Buib (Bubulcus ibis); Capl (Caracara plancus); Caru (Cathartes aura); Chto (Chauna torquata); Chci (Chroicocephalus cirrocephalus); Chma (Chroicocephalus maculipennis); Chru (Chrysomus ruficapillus); Cofl (Coereba flaveola); Coca (Colaptes campestris); Come (Colaptes melanochlorus); Coli (Columba livia); Copi (Columbina picui); Cota (Columbina talpacoti); Coat (Coragyps atratus); Cygu (Cyclarhis gujanensis); Devi (Dendrocygna viduata); Egth (Egretta thula); Fasp (Falco sparverius); Flfu (Florisuga fusca); Furu (Furnarius rufus); Gaga (Gallinula galeata); Gugu (Guira guira); Hime (Himantopus melanurus); Hycr (Hylocharis crhysura); Icca (Icterus cayanensis); Jaja (Jacana jacana); Leve (Leptotila verreauxi); Mari (Machetornis rixosa); Misa (Mimus saturninus); Micma (Milvago chimachima); Micgo (Milvago chimango); Mymo (Myiopsitta monachus); Paco (Paroaria coronata); Papi (Setophaga pitiayumi); Phsi (Phaetusa simplex); Phin (Phimosus infuscatus); Phve (Phylloscartes ventralis); Pibo (Pipraeidea bonariensis); Pisu (Pitangus sulphuratus); Plch (Plegadis chihi); Prta (Progne tapera); Psvi (Pseudoleistes guirahuro); Pycy (Pygochelidon cyanoleuca); Sifl (Sicalis flaveola); Sysi (Syrigma sibilatrix); Tale (Tachycineta leucorrhoa); Thbo (Molothrus bonariensis); Tasa (Tangara sayaca); Trmu (Troglodytes musculus); Tuam (Turdus amaurochalinus); Turu (Turdus rufiventris); Tysa (Tyrannus savana); Vach (Vanellus chilensis); Xoir (Xolmis irupero); Zeau (Zenaida auriculata); Zoca (Zonotrichia capensis). Variáveis ambientais: Arv (número de árvores), at2 (número de construções de até dois andares), m2 (número de construções de mais de dois andares), A.Aq (presença de ambiente aquático), A.ab (presença de ambiente aberto)].

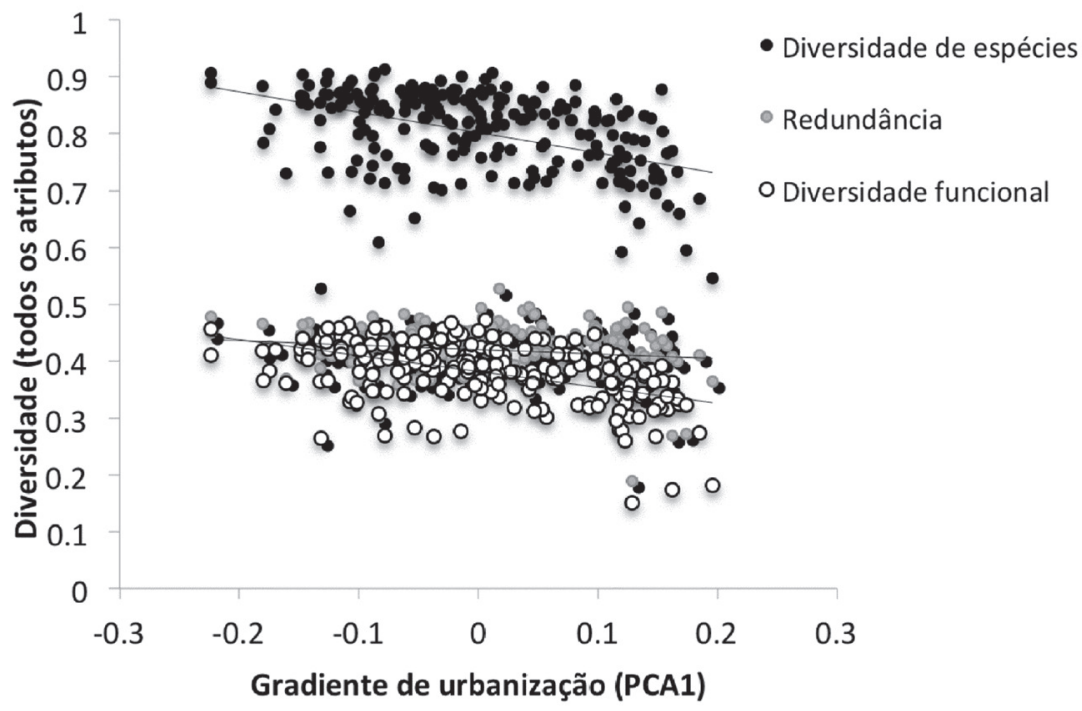

Fig. 4. Influência da intensidade de urbanização sobre a diversidade e redundância funcional, bem como diversidade taxonômica, baseado na totalidade dos atributos das aves registradas na cidade de Pelotas, RS, Brasil entre outubro de 2007 a setembro de 2008. 
a)

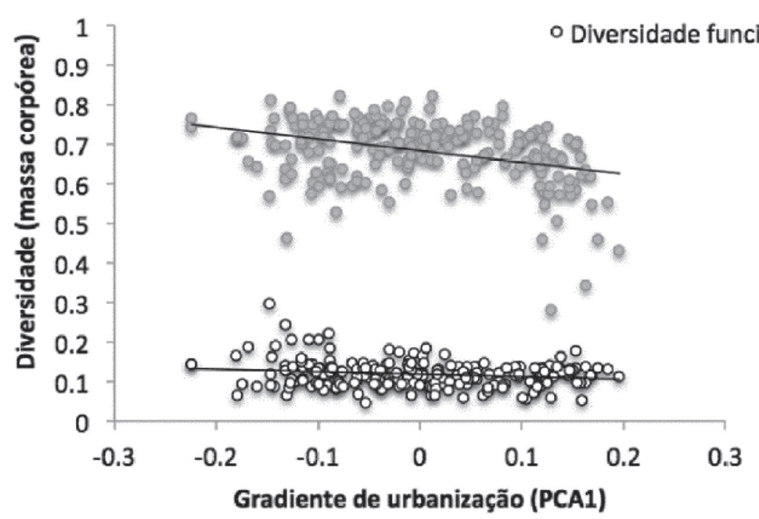

c)

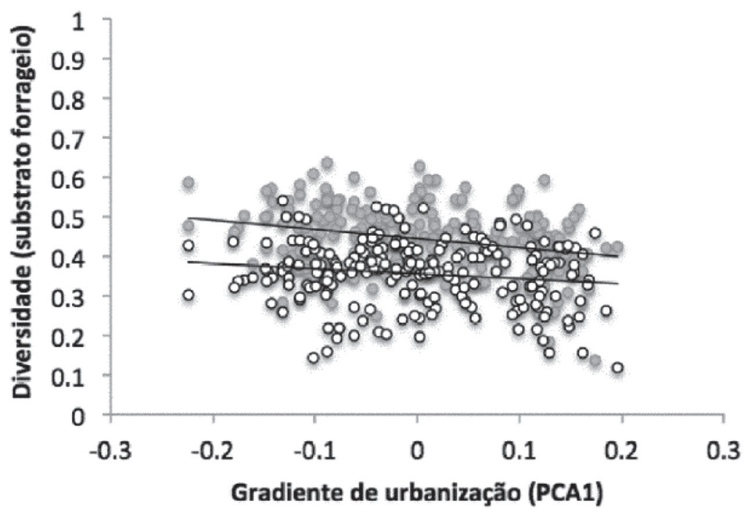

b)

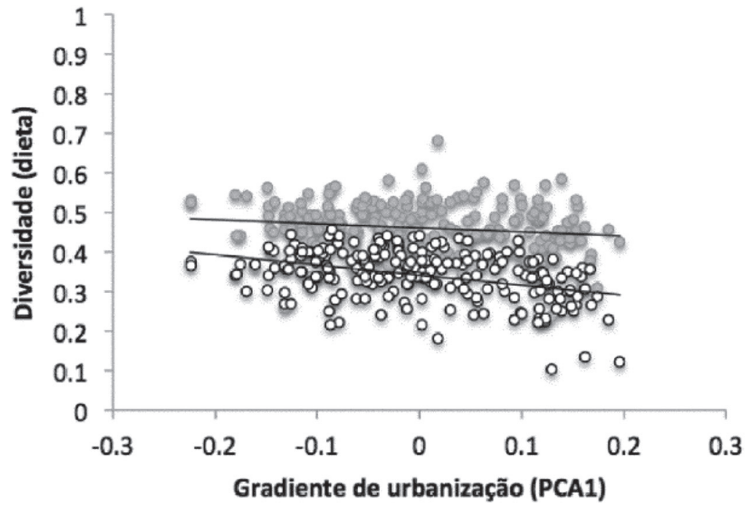

d)

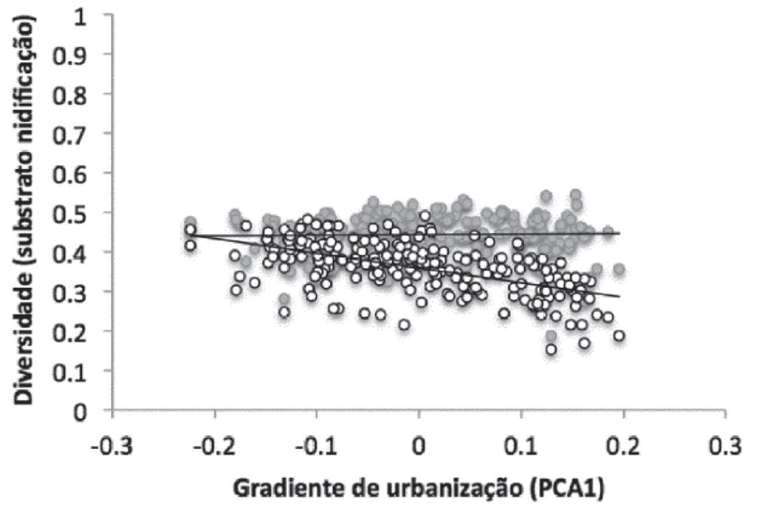

Fig. 5. Influência da intensidade de urbanização sobre a diversidade e redundância funcional, levando em consideração os atributos de massa corpórea (a), tipo de dieta (b), substrato de forrageio (c) e de nidificação (d) das aves registradas na cidade de Pelotas, RS, Brasil entre outubro de 2007 a setembro de 2008.

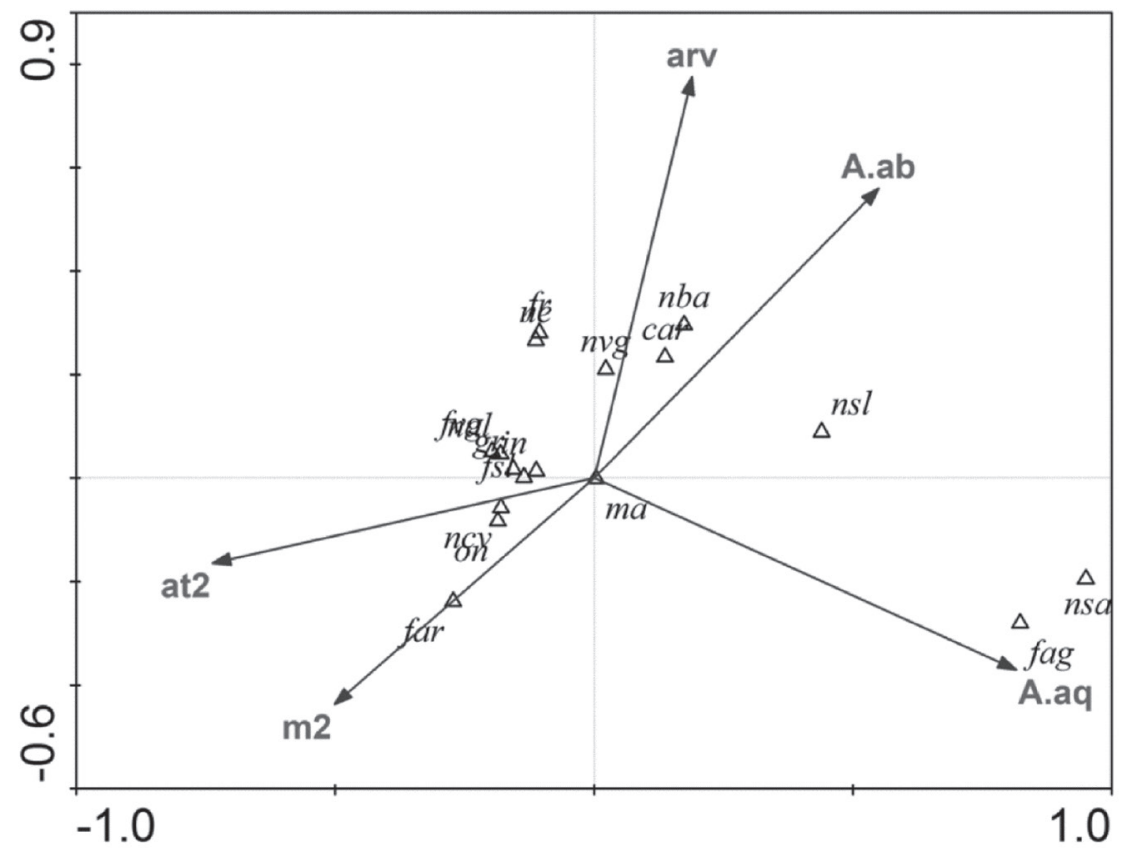

Fig. 6. Diagrama de ordenação definido por análise de correspondência canônica (CCA), relacionado a composição funcional (atributos) de aves com as variáveis urbanas. As setas representam as variáveis ambientais e os triângulos representam os atributos funcionais (Atributos: ma, massa; on, onívoro; car, carnívoro; in, insetívoro; Gr, granívoro; fr, frugívoro; NE, nectarívoro; fsl, forrageamento no solo; fag, forrageamento na água; far, forrageamento no ar; fvg, forrageamento na vegetação; ncv, ninho em cavidades; nvg, ninho em vegetação; nsl, ninho em solo; nsa, ninho sobre a água; nal, ninho em locais altos; nba, ninhos em locais baixos. Variáveis: arv, número de árvores; at2, número de construções de até dois andares; m2, número de construções de mais de dois andares; A.ab, presença de ambiente aberto; A.aq, presença de ambiente aquático). 


\section{DISCUSSÃO}

Os efeitos negativos da urbanização sobre a avifauna ocorrem em nível global e têm sido demonstrados principalmente em cidades com mais de um milhão de habitantes (MCKInNEY, 2002; VILlegas \& GaRITANOZavala, 2010; Conole \& Kirkpatrick, 2011; Fontana et al., 2011; Pellissier et al., 2012). No entanto, nossos resultados mostraram que estes efeitos são sentidos mesmo em uma cidade considerada média, sendo possível observar a existência de um gradiente de intensidade de urbanização que causa respostas evidentes na avifauna. No Brasil, as cidades consideradas médias apresentam as maiores taxas de crescimento populacional e consequente aumento de urbanização (IPEA, 2008) e devem ser consideradas prioritárias para a realização de estudos dos efeitos da urbanização sobre a biodiversidade (LuCK et al., 2013).

Tanto a riqueza como a abundância diminuíram à medida que a intensidade de urbanização aumenta. Este padrão tem sido observado para riqueza (DíAz \& ARMESTO, 2003; LeVEAU \& LeVEAU, 2004; Villegas \& GaritanoZavala, 2010), contudo, a maioria dos trabalhos indica que a abundância é mais elevada em níveis intermediários de urbanização (CHACE \& WALSH, 2006). Nossos resultados - redução da abundância com aumento da intensidade de urbanização - podem decorrer da exclusão de Passer domesticus das contagens. Por ser uma espécie muito abundante, principalmente em áreas peridomiciliares (SICK, 1997), sua inclusão poderia elevar a abundância nos níveis intermediários de urbanização, gerando um padrão similar ao observado em outros estudos (CHACE \& WALSH, 2006). Outro fator pode ter sido a extensão do gradiente estudado, ou seja, áreas exclusivamente rurais não foram amostradas. Portanto, o extremo menos urbanizado neste estudo, o qual apresentou maiores valores de abundância, seria o nível intermediário de urbanização do gradiente.

Os resultados apontaram que o gradiente de urbanização estudado explicou cerca de $20 \%$ para ambas as variáveis - riqueza e abundância - indicando que outros elementos existentes no ambiente urbano, e que não foram avaliados, são importantes, tais como composição da vegetação nativa (VILLEGAS \& GARITANO-ZAVALA, 2010) ou níveis de poluição sonora (FonTANA et al., 2011). Ainda assim, as variáveis urbanas mensuradas demonstraram ser boas indicadoras do gradiente de intensidade de urbanização, uma vez que foi possível observar sua influência tanto em parâmetros taxonômicos quanto funcionais das aves.

A arborização, o número de construções e a presença de áreas úmidas foram variáveis que influenciaram na composição da avifauna de Pelotas. Nossos resultados identificaram uma mudança na composição da avifauna à medida que o número de árvores ou a proporção de áreas com vegetação aberta aumenta. Isto pode estar relacionado com a escassez de parques e praças no centro urbano de Pelotas, que possui um entorno bastante diversificado, composto por um mosaico de diferentes paisagens (plantações de arroz, silvicultura, ambientes lacustres extensos, criação de gado) (SACCO et al., 2013). A arborização é um dos fatores que mais influencia na chegada e na possibilidade de permanência das espécies nas cidades (BLAIR \& LAUNER, 1997), oferecendo locais para nidificação, abrigo, descanso e fontes de recursos alimentares (SAVARD et al., 2000; FERNÁNDEZ-JURICIC \& JokimäKI, 2001; Melles et al., 2003; Toledo et al., 2012), usualmente gerando uma resposta positiva na avifauna (MArzluff et al., 2001; McKinney, 2002). Em Pelotas, a arborização teve influência importante para a presença de espécies que habitam florestas secundárias como, por exemplo, Coereba flaveola (Linnaeus, 1758), Hylocharis chrysura (Shaw, 1812), ou Tyrannus savana (Vieillot, 1808). O efeito do número de construções sobre a avifauna provavelmente se deve à diminuição da disponibilidade de recursos em função do aumento do desenvolvimento urbano (Melles et al., 2003). Espécies como Columbina picui (Temminck, 1813), Pitangus sulphurathus, Pygochelidon cyanoleuca (Vieillot, 1817), Troglodytes musculus (Naumann, 1823) se mostraram ligadas a variáveis de urbanização, indicando que toleram mais este tipo de ambiente. Em Pelotas - localizada numa região de área úmida no Bioma Pampa - cerca $61 \%$ dos pontos de amostragem continham presença de áreas abertas e em $25 \%$ havia algum tipo de ambiente aquático presente. Como esperado, espécies características de áreas úmidas se posicionaram no lado oposto ao das áreas caracterizadas pela maior intensidade de urbanização, indicando um efeito negativo da urbanização sobre a avifauna limícola/ aquática. Em centros urbanos a pressão sobre áreas úmidas ocorre devido à realização de drenagem e aterramentos para ampliação de áreas urbanas e a retirada de água para abastecimento da população (CARVALHO \& OzÓRIO, 2007). Como resultado, a movimentação de animais se torna mais difícil e recursos essenciais podem se tornar inacessíveis, com uma provável alteração na composição e estrutura da comunidade.

A diversidade funcional total e a diversidade taxonômica diminuiram com a intensidade de urbanização, enquanto observou-se uma diminuição sutil na redundância funcional. Neste sentido, nosso estudo indica uma perda na funcionalidade com a urbanização, além de uma possível homogeneização biótica da avifauna à medida que os níveis de urbanização se tornam mais elevados (LUCK \& Smallbone, 2011), em função da menor riqueza e a possível semelhança na composição entre ambientes urbanos. Considerando que a redundância dentre as espécies presentes permanece pouco alterada ao longo do gradiente de urbanização, a menor diversidade funcional reflete, de fato, a ausência de aves com características específicas. Da mesma forma, Petchey et al. (2007), avaliando várias assembléias de aves no Reino Unido, encontraram níveis baixos de redundância funcional a despeito de locais com menor diversidade funcional, indicando uma alteração direcional das mudanças na composição destas assembléias. Nas áreas de menor urbanização, a proporção de aves funcionalmente redudantes é maior, o que garante uma 
certa segurança na manutenção de determinadas funções ecológicas (NAEEM, 1998). Como discutido por OLDEN et al. (2004), a redução na redundância funcional é causada pelo empobrecimento das espécies que constituem as comunidades em situações de distúrbios severos, tal como a urbanização, que somado a perda de determinados atributos funcionais, leva à homogeneização biótica.

Padrões de diversidade funcional sempre refletem o conjunto de atributos selecionados (Mouchet et al., 2010) e, portanto, frente a um conjunto grande de características, é fundamental avaliar variações mais específicas. Considerando apenas o substrato de nidificação, a diversidade funcional se mostrou a mais afetada pela intensidade de urbanização, mesmo resultado obtido por Vignoli et al. (2013). Uma vez que diminuem as possibilidades de locais para nidificação (LIM \& SodHI, 2004), espécies que fazem ninhos em vegetação, em locais baixos e no solo foram mais abundantes em locais menos urbanizados e positivamente relacionados ao número de árvores, à proporção de áreas abertas e à presença de ambientes aquáticos. Por outro lado, a proporção de aves que fazem ninhos altos e em cavidades é maior quanto maior a intensidade de urbanização. A falta de arborização e o aumento da edificação nas áreas com maior intensidade de urbanização certamente refletem este padrão (LANCASTER \& ReEs, 1979; Croci et al., 2008; ConOle \& KirkPatrick, 2011; SACCO et al., 2013), assim como interferência direta dos seres humanos e a pressão de predação (CROCI et al., 2008; LóPEZ-Flores et al., 2009). Espécies que preferem nidificar em locais baixos e no solo estão mais suscetíveis à predação pelos animais domésticos, como cães e gatos (Crocr et al., 2008), enquanto que ninhos em cavidades ou locais elevados provavelmente são mais protegidos destas ameaças.

A relação negativa entre o tamanho das espécies em relação à massa - e a intensidade de urbanização que observamos é oposta ao padrão encontrado em outros estudos (GonzÁLEZ OreJA et al., 2007; KARK et al., 2007; Croci et al., 2008; CONOLE \& KIRKPATRICK, 2011). Ou seja, enquanto em Pelotas as aves tenderam a ser maiores em níveis baixos de urbanização, em outras regiões as aves tenderam a ser menores. Essa relação provavelmente se deve as características da paisagem de cada região. Em áreas urbanas cujas regiões são predominantemente florestais as espécies de aves tendem a ser menores se comparadas as de ambientes abertos. O pool regional de espécies da região do presente estudo apresenta uma grande proporção de aves de habitats aquáticos e campestres, que tendem a ter massas corpóreas maiores (MäHLER JR. et al., 1996). Como mencionado anteriormente, as espécies de habitats aquáticos e campestres foram negativamente associadas com a intensidade da urbanização, refletindo esse padrão de espécies maiores em áreas menos urbanizadas.

A diversidade funcional dos atributos de dieta diminuiu com o gradiente de urbanização, sendo a redundância pouco afetada, indicando perda de funcionalidade, resultado semelhante obtido por PAUw \&
Louw (2012) em áreas urbanizadas na África do Sul. A onivoria foi o atributo relacionado à dieta predominante em áreas mais urbanizadas, resultado comum encontrado em outros estudos (tais como LANCASTER \& REes, 1979; Clergeau et al., 1998; Jokimäki \& Suhonen, 1998; KARK et al., 2007; Croci et al., 2008; Conole \& KirKPATRICK, 2011; VignOLI et al., 2013). As espécies onívoras apresentam maior vantagem nas cidades, onde existe uma maior variedade de itens, já que os humanos produzem estes recursos, inclusive o lixo (CLERGEAU et al., 1998). Carnívoros foram mais presentes em áreas menos urbanizadas, provavelmente pelo fato de nestas áreas existirem ambientes abertos que beneficiam os mesmos devido as suas grandes áreas de vida (SICK, 1997).

Segundo Robinson \& Holmes (1982), o comportamento de forrageamento das aves é influenciado pela complexidade estrutural do ambiente e de seu entorno. Em nosso estudo, espécies com comportamento de forrageamento no ar foram favorecidas pela intensidade de urbanização, estando associadas a construções de mais de dois andares. Possivelmente em áreas mais urbanizadas existe maior disponibilidade de poleiros em diferentes alturas, o que pode beneficiar técnicas onde a ave se lança no ar a partir de um poleiro para capturar a presa (SICK, 1997). As áreas mais arborizadas, que também ofereceriam poleiros para forrageamento no ar, não estiveram associadas a este tipo de forrageio, talvez por não conseguirem atingir alturas obtidas pelas construções. Já o forrageamento na água esteve ligado aos ambientes aquáticos em áreas pouco urbanizadas. Considerando estas diferenças e o fato que os índices de diversidade e redundância calculados para este conjunto de atributos foram pouco influenciados pelo gradiente de urbanização, podemos inferir que esteja ocorrendo uma substituição destes atributos conforme o gradiente de urbanização.

Os resultados deste estudo evidenciam, além dos efeitos negativos da urbanização sobre a riqueza e a composição da avifauna, os efeitos sobre a diversidade e composição funcional da assembleia de aves, evidenciando uma perda funcional mesmo em uma cidade considerada de médio porte. Pôde-se obervar que na área urbana estudada os "tolerantes urbanos" se diferenciaram dos "intolerantes urbanos" em relação à dieta (onívoros versus carnívoros), ao substrato de forrageamento (forrageio no ar versus forrageio na água) e em relação ao substrato de nidificação (intolerantes forrageiam na vegetação, em locais baixos, no solo e sobre a água). Ao mesmo tempo, foi possível observar a importância da arborização e da presença de ambientes aquáticos ou abertos para o aumento da riqueza, da abundância e da diversidade funcional, evidenciando a relevância da disponibilidade e conservação de diversos tipos de habitat mesmo em sistemas urbanos. Tendo em vista que a intensidade dos efeitos das características ambientais varia entre espécies e comunidades (ORTEGAÁlvarez \& MACGregor-Fors, 2009), diferenças no efeito da urbanização podem estar ligadas com o contexto regional da área urbana. No presente estudo, a área urbana 
se insere num contexto de áreas úmidas (Planície Costeira) do Bioma Pampa e, portanto, características associadas a disponibilidade de água e vegetação aberta foram de extrema relevância para a manutenção de características taxonômicas e funcionais da assembleia de aves e, consequentemente, sua conservação.

Agradecimentos. Aos Drs. Oswaldo Marçal Jr., Carla S. Fontana e Felipe Zilio pelas críticas e sugestões. Ao Dr. Ronei Baldissera pelo auxílio na confecção das figuras. À Coordenação de Aperfeiçoamento de Pessoal de Nível Superior (CAPES) pela concessão de bolsa de mestrado à primeira autora. Ao $\mathrm{CNPq}$ pelas bolsas de produtividade de Sandra Cristina Müller (307719/2012-0) e Sandra Maria Hartz (306816/2010-5).

\section{REFERÊNCIAS BIBLIOGRÁFICAS}

Alberti, M.; Botsford, E. \& Cohen, A. 2001. Quantifying the urban gradient: linking urban planning and ecology. In: MARZLUFF, J.; Bowman, R. \& DonnelLy, R. eds. Avian ecology and conservation in an urbanizing world. Norwell, Kluwer Academic, p.89-115.

Anjos, L. 2001. Bird communities in five Atlantic Forest fragments in southern Brazil. Ornitologia Neotropical 12:11-27.

Belton, W. 1994. Aves do Rio Grande do Sul. Distribuição e Biologia. São Leopoldo, Unisinos. 584p.

Bibby, C.; Burgess, N. \& Hill, D. 1992. Bird census techniques. London, Academic Press. 280p.

BLAIR, R. 1996. Land use and avian species diversity along an urban gradient. Ecological Applications 6:506-519.

Blair, R. \& LAUner, A. 1997. Butterfly diversity and human land use: species assemblages along an urban gradient. Biological Conservation 80:113-125.

Calliari, L. 1998. Características geomorfológicas. In: Seeliger, U.; Odebrecht, C. \& Castello, J. eds. Os ecossistemas costeiro e marinho do extremo sul do Brasil. Rio Grande, Ecoscientia, p.101-104.

Carvalho, A. \& Ozorio, C. 2007. Avaliação sobre os banhados do Rio Grande do Sul, Brasil. Revista Ciência e Ambiente 1:83-95.

CBRO - Comitê Brasileiro de Registros OrnitolóGicos. 2014. Lista das aves do Brasil. 11 $1^{\mathrm{a}}$. ed. Disponível em $<\mathrm{http}$ ://www.cbro.org.br>.

CHACE, J. \& WALSH, J. 2006. Urban effects on native avifauna: a review. Landscape Urban Planning 74:46-69.

Clergeau, P.; Savard, P.; Mennechez, G. \& Falardeau, G. 1998. Bird abundance and diversity along an urban-rural gradient: a comparative study between two cities on different continents. Condor 100:413425.

CONOLE, L. \& KIRKPATRICK, J. 2011. Functional and spatial differentiation of urban bird assemblages at the landscape scale. Landscape Urban Planning 100:11-23.

Croci, S.; Butet, A. \& Clergeau, P. 2008. Does urbanization filter birds on the basis of their biological traits. Condor 110:223-240.

de Bello, F.; LePS, J.; Lavorel, S. \& Moretti, M. 2007. Importance of species abundance for assessment of trait composition: an example based on pollinator communities. Community Ecology 8:163-170.

Del hoyo, J.; Elliot, A. \& Christie, D. 2003-2006. Handbook of the birds of the world (Broadbills to tapaculos, vols. 8-11). Barcelona, Lynx. 845p.

Del hoyo, J.; Elliot, A. \& Sargatal, J. 1992-2002. Handbook of the birds of the world (Ostrich to ducks, vols. 1-7). Barcelona, Lynx. 696p.

DíAz, I. \& Armesto, J. 2003. La conservación de las aves silvestres en ambientes urbanos de Santiago. Revista Ambiente y Desarollo de CIPMA 19:31-38.

DonNelly, R. \& MARZlufF, J. 2006. Relative importance of habitat quantity, structure, and spatial pattern to birds in urbanizing environments. Urban Ecosystems 9:99-117.

FERNÁNDEZ-JuRICIC, E. \& JOKIMÄKI, J. 2001. A habitat island approach to conserving birds in urban landscapes: case studies from southern and northern Europe. Biodiversity and Conservation 10:2023-2043.
Flynn, D.; Gogol-Prokurat, M.; Nogeire, T.; Molinari, N.; Richers, B.; Lin, B.; Simpson, N.; MAYField, M. \& DeClerCK, F. 2009. Loss of functional diversity under land-use intensification across multiple taxa. Ecology Letters 12:22-33.

Fontana, C.; Burger, M. I. \& Magnusson, W. 2011. Bird diversity in a subtropical South-American City: effects of noise levels, arborisation and human population density. Urban Ecosystems 14:341-360.

GarafFa, P.; Filloy, M. \& BellocQ, I. 2009. Bird community responses along urban-rural gradients: Does the size of the urbanized area matter? Landscape Urban Planning 90:33-41.

Gilbert, O. 1989. The Ecology of Urban Habitats. London, Chapman \& Hall. 369p.

González Oreja, J.; Regidor, C.; Franco, D.; Ordaz, A. \& Satín, L. 2007. Caracterizatión ecológica de la avifauna de los parques urbanos de la ciudad de Puebla (México). Ardeola 54:53-67.

Gower, J. 1971. A general coefficient of similarity and some of its properties. Biometrics 27:857-872.

Hahs, A. \& MCDonnell, M. 2006. Selecting independent measures to quantify Melbourne's urban-rural gradient. Landscape Urban Planning 78:435-448.

IBGE - FundaÇÃo Instituto Brasileiro de Geografia e Estatística. 1992. Manual técnico da vegetação brasileira. Rio de Janeiro, Série Manuais Técnicos em Geociências, n.1.92p.

2010. Contagem da população 2010 - Pelotas/RS. Disponível em $<$ http://www.ibge.gov.br $>$. Acessado em 22.05.2012.

IPEA - Instituto de Pesquisa Econômica e APlicada. 2008. População e PIB das cidades médias crescem mais que no resto do Brasil. Disponível em $<\mathrm{http}: / /$ www.redbcm.com.br/arquivos /bibliografia/ pesquisa\%20ipea.pdf $>$. Acessado em 13.04.2010

JokimäKI, J. \& SUHONEN, J. 1998. Distribution and habitat selection of wintering birds in urban environments. Landscape and Urban Planning 39:253-263.

Kark, S.; Iwaniuk, A.; Schalimtzek, A. \& Banker, E. 2007. Living in the city: can anyone become an 'urban exploiter'? Journal of Biogeography 34:638-651.

KRÜGEL, M. \& ANJos, L. 2000. Bird communities in forest remnants in the city of Maringa, Paraná State, Southern Brazil. Ornitologia Neotropical 11:315-330.

LanCASTER, R. \& ReEs, W. 1979. Bird communities and the structure of urban habitats. Canadian Journal of Zoology 57:2358-2368.

Leveau, L. \& Leveau, C. 2004. Comunidades de aves en un gradiente urbano de la ciudad de Mar del Plata, Argentina. Hornero 19:13-21.

Lim, H. \& SodHI, N. 2004. Responses of avian guilds to urbanization in a tropical city. Landscape and Urban Planning 66:199-215.

LóPEZ-Flores, V.; MACGREGor-Fors, I. \& SCHONDUBE, J. 2009. Artificial nest predation along a neotropical urban gradient. Landscape and Urban Planning 92:90-95.

LuCK, G. \& SMALLBONE, L. 2011. The impact of urbanization on taxonomic and functional similarity among bird communities. Journal of Biogeography 38:894-906.

Luck, G.; Smallbone, L. \& Sheffield, K. 2013. Environmental and socio-economic factors related to urban bird communities. Austral Ecology 38:111-120.

MäHLER JR., J.; KINDEL, A. \& Kindel, E. 1996. Lista comentada das espécies de aves da Estação Ecológica do Taim, Rio Grande do Sul, Brasil. Acta Biologica Leopoldensia 18:69-113.

Mantel, N. 1967. The detection of disease clustering and a generalized regression approach. Cancer Research 27:209-220.

MARZLUfF, J. 2001. Worldwide urbanization and its effects on birds. In: Marzluff, J.; Bowman, R. \& Donnelly, R. eds. Avian ecology and conservation in an urbanizing world. Boston, Kluwer Academic, p.19-47.

Marzluff, J.; Bowman, R. \& Donnelly, R. 2001. Avian ecology and conservation in an urbanizing world. Boston, Kluwer Academic Pub., 585p.

MARZLUFF, J. \& EWING, K. 2001. Restoration of fragmented landscapes for the conservation of birds: a general framework and specific recommendations for urbanizing landscapes. Restoration Ecology 9:280-292.

MCKInNEY, M. 2002. Urbanization, biodiversity and conservation. Bioscience 52:883-890. 
McKinney, M. \& Lockwood, J. 1999. Biotic homogenization: a few winners replacing many losers in the next mass extinction. Trends in Ecology and Evolution 14:450-453.

Melles, S.; Glenn, S. \& Martin, K. 2003. Urban bird diversity and landscape complexity: species-environment associations along a multiscale habitat gradient. Conservation Ecology 7(1). Disponível em <http://www.consecol.org/vol7/iss1/art5>. Acessado em 15.05.2012.

Micheli, F. \& Halpern, B. 2005. Low functional redundancy in coastal marine assemblages. Ecology Letters 8:391-400.

Mouchet, M.; VilléGer, S.; Mason, N. \& Mouillot, D. 2010. Functional diversity measures: an overview of their redundancy and their ability to discriminate community assembly rules. Functional Ecology 24:867-876.

NAEEM, S. 1998. Species redundancy and ecosystem reliability. Conservation Biology 12:39-45.

Olden, J.; LeRoy Poff, N.; Douglas, M.; Douglas, M. \& Fausch, K. 2004. Ecological and evolutionary consequences of biotic homogenization. Trends in Ecology and Evolution 19:18-24.

Ortega-Álvarez, R. \& MacGregor-Fors, I. 2009. Living in the big city: Effects of urban land-use on bird community struture, diversity, and composition. Landscape and Urban Planning 90:189-195.

PAuw, A. \& Louw, K. 2012. Urbanization drives a reduction in functional diversity in a guild of nectar-feeding birds. Ecology and Society 17. Disponível em <http://www.ecologyandsociety.org/vol17/iss2/ art27>. Acessado em 15.12.2012.

Pellissier, V.; Cohen, M.; Boulay, A. \& Clergeau, P. 2012. Birds are also sensitive to landscape composition and configuration with the city centre. Landscape and Urban Planning 104:181-188.

Petchey, O. \& Gaston, K. 2002. Functional diversity (FD), species richness and community composition. Ecology Letters 5:402-411.

Petchey, O.; Evans, K.; Fishburn, I. \& Gaston, K. 2007. Low functional diversity and no redundancy in British avian assemblages. Journal of Animal Ecology 76:977-985.

Petchey, O.; O’Gorman, E. \& Flynn, D. 2009. A functional guide to functional diversity measures. In: NAEeM, S.; Bunker, D.; НеCtor, A.; Loreua, M. \& Perrings, C. eds. Biodiversity and Human Impacts. Oxford, Oxford University Press, p.49-59.

Pillar, V. 2006. MULTIV: Multivariate exploratory analisys, randomizating testing and boostraping resampling, users guide. v.2.4, Porto Alegre, Universidade Federal do Rio Grande do Sul. Disponível em: $<$ http://ecoqua.ecologia.ufrgs.br/arquivos/Software/ Multiv>.

Pillar, V.; Duarte, L.; Sosinski, E. \& Joner, F. 2009. Discriminating trait-convergence and trait-divergence assembly patterns in ecological community gradients. Journal of Vegetation Science 20:334-348.

Pillar, V.; Blanco, C.; Müller, S.; Sosinski, E.; Joner, F. \& Duarte, L. 2013. Functional redundancy and stability in plant communities. Journal of Vegetation Science 24:963-974.
PROBIO - Ministério do Meio Ambiente. 2007. Cobertura Vegetal do Bioma Pampa - Relatório Técnico. Porto Alegre, Universidade Federal do Rio Grande do Sul. Disponível em <http://www.ecologia. ufrgs.br/labgeo $>$. Acessado em 22.07.2011.

RAO, C. 1982. Diversity and dissimilarity coefficients - a unified approach. Theoretical Population Biology 21:24-43.

Robinson, S. \& Holmes, R. 1982. Foraging behavior of forest birds: the relationships among search tactics, diet, and habitat structure. Ecology 63:1918-1931.

Sacco, A.; Bergman, F. \& Rui, A. 2013. Assembleia de aves na área urbana do município de Pelotas, Rio Grande do Sul, Brasil. Biota Neotropica 13. Disponível em <http://www.biotaneotropica.org.br/ v13n2/en/abstract?inventory+bn01113022013>.

Savard, J.-P.; Clergeau, P. \& Mennechez, G. 2000. Biodiversity concepts and urban ecosystems. Landscape and Urban Planning 48:131-142.

SICK, H. 1997. Ornitologia Brasileira. Rio de Janeiro, Editora Nova Fronteira. 912p.

Stevens, R.; Cox, S.; Strauss, R. E. \& Willig, M. 2003. Patterns of functional diversity across an extensive environmental gradient: vertebrate consumers, hidden treatments and latitudinal trends. Ecology Letters 6:1099-1108.

Stotz, D.; Fitzpatrick, J.; Parker iII, T. \& Moskovits, D. 1996. Neotropical Birds: ecology and conservation. Chicago, University of Chicago Press. 502p.

Vignoli, L; Scirè, S. \& Bologna, M. 2013. Rural-urban gradiente and land use in a millenary metropolis: how urbanization affects avian functional groups and the role of old villas in bird assemblage patterning. Web Ecology 13:49-67.

Ter BrAAK, C. 1986. Canonical correspondence analysis: a new eigenvector technique for multivariate direct gradient analysis. Ecology 67:1167-1179.

Toledo, M.; Donatelli, R. \& Batista, G. 2012. Relation between green spaces and bird community structure in an urban area in Southeast Brazil. Urban Ecosystems 15:111-131.

Vandewalle, M.; De Bello, F.; Berg, M.; Bolger, T.; Dolédec, S.; Dubs, F.; Feld, C.; Harrington, R.; Harrison, P.; Lavorel, S.; Da Silva, P.; Moretti, M.; Niemelä, J.; Santos, P.; Sattler, T.; Sousa, J.; Sykes, M.; Vanbergen, A. \& Woodcock, B. 2010. Functional traits as indicators of biodiversity response to land use changes across ecosystems and organism. Biodiversity and Conservation 19:2921-2947.

Villegas, M. \& Garitano-ZaVAla, A. 2010. Bird community responses to different urban conditions in La Paz, Bolivia. Urban Ecosystems 13:375-391.

Wilcox, B. \& Murphy, D. 1985. Conservation strategy: the effects of fragmentation on extinction. American Naturalist 125:879-887. 